\title{
Serum Cystatin C Concentrations in Patients with Graves' Ophthalmopathy
}

\author{
Nagehan Can ${ }^{1}$, Ercan Ozsoy ${ }^{2}$, Sabiha Gungor Kobat ${ }^{3}$, Elif Yusufoglu ${ }^{3}$, Nevin Ilhan ${ }^{4}$, Tamer Demir ${ }^{5}$ \\ ${ }^{1}$ Department of Ophthalmology, Torbali State Hospital, Izmir, Turkey \\ ${ }^{2}$ Department of Ophthalmology, Haseki Training and Research Hospital, University of Health Sciences, Istanbul, Turkey \\ ${ }^{3}$ Department of Ophthalmology, Elazig Training and Research Hospital, University of Health Sciences, Elazıg, Turkey \\ ${ }^{4}$ Department of Biochemistry, Firat University School of Medicine, Elazıg, Turkey \\ ${ }^{5}$ Department of Ophthalmology, Canakkale Onsekiz Mart University School of Medicine, Canakkale, Turkey
}

Purpose: To evaluate the concentration of serum cystatin C (CysC) in patients with Graves' ophthalmopathy (GO) and the usability of the serum CysC concentrations in the follow-up of the disease.

Methods: Thirty patients with GO and 30 healthy age-matched volunteers were included in this cross-sectional study. GO was diagnosed based on the European Group on Graves' Orbitopathy consensus. Serum thyroid-stimulating hormone, free triiodothyronine, free thyroxine, and $\mathrm{Cys} C$ concentrations were measured in the participants. The serum CysC concentrations were compared between patients with GO and controls. Patients with GO were subdivided into hyperthyroid and euthyroid patients, and their serum CysC concentrations were compared. In addition, the CysC concentrations in hyperthyroid and euthyroid patients with GO were compared separately with those of healthy subjects. Kruskal-Wallis test and Student's $t$-test were used for statistical evaluation.

Results: The mean serum CysC concentrations in $\mathrm{GO}$ patients and controls were $1.04 \pm 0.36$ and $0.74 \pm 0.09 \mathrm{mg} / \mathrm{L}$, respectively. There was a statistically significant difference in the serum CysC concentrations between patients with GO and control subjects ( $p$ $<0.001)$. Fifteen patients had hyperthyroid status, and 15 patients had euthyroid status. The mean serum CysC concentrations in hyperthyroid and euthyroid patients with GO were $1.35 \pm 0.22$ and $0.72 \pm 0.13 \mathrm{mg} / \mathrm{L}$, respectively. Serum CysC concentrations were significantly higher in hyperthyroid patients than in euthyroid patients $(p=0.001)$. In addition, hyperthyroid patients had significantly higher serum CysC concentrations than healthy subjects. Among patients with GO, 21 and nine had mild and moderate-to-severe GO, respectively. Active and inactive $\mathrm{GO}$ were observed in eight and 22 patients, respectively.

Conclusions: The serum CysC concentrations in hyperthyroid patients were higher than those in healthy subjects. Moreover, hyperthyroid patients had higher serum CysC concentrations than euthyroid patients. Further studies with a larger sample size are needed to confirm these results.

Key Words: Cystatin C, Exophthalmos, Serum, Thyroxine, Triiodothyronine

Received: January 23, 2020 Final revision: April 9, 2020 Accepted: April 23, 2020

Corresponding Author: Ercan Ozsoy, MD. Department of Ophthalmology, Haseki Training and Research Hospital, University of Health Sciences, Aksaray Adnan Adivar Street, Fatih, 34130, Istanbul, Turkey. Tel: 90-212-529-4400, Fax: 90-212-589-6229, E-mail: ercanozy@hotmail.com

This manuscript was presented at the 50th National Congress of the Turkish Ophthalmological Association in Antalya. 
Graves' disease (GD) is an organ-specific autoimmune disorder that results from overstimulation of the thyroid gland by the thyroid-stimulating hormone (TSH) receptor antibody [1]. It is the most common reason for hyperthyroidism and affects women (3\%) more commonly than men $(0.5 \%)[2,3]$. GD is characterized by hyperthyroidism, diffuse goiter, ophthalmopathy, and mucous edema in the anterior tibia [4]. Graves' ophthalmopathy (GO) is diagnosed in approximately $25 \%$ to $50 \%$ of patients with GD [5]. It is an autoimmune condition caused by dysfunctions of lymphocytes, autoantibodies, and cytokines. Inflammatory swelling and lymphocytic infiltration of the orbital tissues, such as the extraocular muscles and fat, increase the volume of the orbital cone, which manifests as symptoms of deep orbital pain, conjunctival congestion, chemosis, photophobia, excessive tearing, proptosis, exposure keratitis, corneal ulceration, palpebral edema, lid retraction, diplopia, optic nerve compression, and even vision loss [6-8]. GO follows a characteristic clinical course, including an initial period of disease exacerbation, a peak severity period, an improvement period, and a static period [9].

Cystatin $\mathrm{C}(\mathrm{CysC})$ is a non-glycosylated $13.36-\mathrm{kDa}$ cationic basic protein consisting of 120 amino acids. It is synthesized at a stable rate by nucleated cells. The serum CysC concentration is not influenced by age, sex, muscle mass, inflammation, or malignancy. Glomerular filtration rate is a major determinant of serum CysC concentration, and the thyroid hormones may have metabolic effects on the serum CysC concentration. Hyperthyroidism increases while hypothyroidism decreases the serum $\mathrm{CysC}$ concentration. Moreover, patients who achieve euthyroid status after a hypothyroid status demonstrate increased serum CysC concentrations [10-13].

Although the correlation between thyroid dysfunction and serum CysC concentration is well established, the association between $\mathrm{GO}$ and serum CysC concentration has not been investigated, to the best of our knowledge. The aims of this study were to evaluate the effects of GO on serum $\mathrm{Cys} C$ concentration and to investigate the possible clinical relevance of serum $\mathrm{CysC}$ concentration in the follow-up of patients with GO.

\section{Materials and Methods}

This cross-sectional study included 30 patients with GO and 30 healthy subjects. The study protocol was approved by the institutional review board (approval number: 08/30). Informed consent was obtained from the participants, and the study was conducted in accordance with the ethical standards outlined in the Declaration of Helsinki. The patients with GO were categorized under the GO group, and the healthy subjects were categorized under the control group. The patients with GO were receiving anti-thyroid medication (methimazole or propylthiouracil) during the study, and they were subclassified as hyperthroid or euthyroid based on their thyroid status. In the GO group, 15 patients had hyperthyroid status and 15 had euthyroid status. Of the GO patients with hyperthyroid status, nine had just initiated antithyroid drugs, and six were noncompliant with the antithyroid therapy (poorly controlled hyperthyroidism). Hyperthyroid status was defined as when patients on anti-thyroid medication had typical symptoms and laboratory data, including increased thyroid hormone concentration and decreased TSH concentration, and euthyroid status was defined when patients on anti-thyroid medication had serum concentrations of free triiodothyronine (FT3) and free thyroxine (FT4) within normal range. Patients with GO were evaluated by the department of endocrinology to exclude a possible misdiagnosis of hyperthyroid or euthyroid status.

Ophthalmological examination was performed to evaluate the following: corneal and conjunctival statuses with the slit-lamp examination, exophthalmos with the Hertel exophthalmometer (Oculus, Wetzlar, Germany), lid aperture in the primary gaze, eye movements, and diplopia with the binocular single-vision test. The presence of spontaneous retrobulbar pain or pain on attempted upward or downward gaze was investigated through patient interview. The exclusion criteria were neurologic diseases, eye diseases (such as an ocular surface disease), strabismus, glaucoma, retinal diseases, optic neuritis, and other diseases known to affect the serum CysC concentration. The control group included individuals who had no systemic or eye diseases.

The amount of ocular protrusion was measured by one person using the same type of Hertel exophthalmometer to avoid intra- and inter-observer variations. Eye protrusion that measured over $21 \mathrm{~mm}$, or an at least $2 \mathrm{~mm}$ difference 
between the eyes was regarded as exophthalmos. The distances of the corneal light reflex from the upper and lower eyelids were measured in the primary gaze.

GO was diagnosed based on the guidelines of the European Group on Graves' Orbitopathy (EUGOGO) consensus. Clinical Activity Score $<3$ and $\geq 3$ were defined as inactive and active GO, respectively. Disease severity was evaluated on the severity scale provided by the EUGOGO [14].

Blood samples were obtained from participants and centrifuged at 3,000 $\times g$ for 10 minutes to separate the sera. Serum CysC concentrations were measured with the nephelometric immunoassay method using the $\mathrm{N}$ Latex cystatin $\mathrm{C}$ assay kit (order no. OQNM13; Dade Behring, Marburg, Germany) on the Siemens Dade Behring BN II Nephelometer (Siemens, Munich, Germany) with a reference interval of $0.62-1.11 \mathrm{mg} / \mathrm{L}$.

Serum TSH, FT3, and FT4 concentrations were measured with a chemiluminescent immunoassay method using the ADVIA Centaur XP system (Siemens Diagnostics, Tarrytown, NY, USA). The reference ranges of TSH, FT3, and FT4 were $0.5-5.5 \mathrm{IU} / \mathrm{mL}, 1.57-4.71 \mathrm{pg} / \mathrm{mL}$, and 0.89 $1.76 \mathrm{ng} / \mathrm{dL}$, respectively.

Statistical evaluation was performed using IBM SPSS Statistics ver. 20.0 (IBM Corp., Armonk, NY, USA). The results are expressed as mean \pm standard deviation. The Kolmogorov-Smirnov test was performed to determine the normality of data distribution. Student's $t$-test and Kruskal-Wallis test were used for the data with and without normal distribution, respectively. In addition, pairwise comparisons were used for a sub-analysis. A $p$-value $<0.01$ was considered statistically significant.

\section{Results}

The GO and control groups included 30 patients each. Table 1 presents the demographic characteristics of the groups. There were no statistically significant differences in the mean age or sex between the groups.

Fifteen patients $(50 \%)$ with GO had a euthyroid state, and 15 patients $(50 \%)$ with GO had a hyperthyroid state. The 60 eyes of 30 patients with GO showed the following results: mean duration of eye involvement of 13.0 months (range, 8-30 months), bilateral involvement in 24 patients $(80 \%)$, a mean Hertel exophthalmometry measurement of
$20.2 \pm 4.1 \mathrm{~mm}$, proptosis in eight eyes (13.3\%), lid retraction in 16 eyes (26.6\%), lid lag in the downgaze in 11 eyes (18.3\%), and lagophthalmos in one eye (1.6\%). In seven eyes $(11.6 \%)$, restricted motility was observed, mostly in the downgaze. Two patients $(6.6 \%)$ had diplopia, while severe corneal problems, optic neuropathy, or vision loss were not seen in any patient.

Based on the severity scale of EUGOGO, 21 (70\%), nine $(30 \%)$, and zero $(0 \%)$ patients had mild, moderate-to-severe, and severe GO, respectively. Eight (26.6\%) and 22 patients (73.3\%) had active and inactive GO, respectively. All patients with euthyroid status had inactive GO (Clinical Activity Score <3). Twenty-six patients $(86.6 \%)$ complained of spontaneous retrobulbar pain and/or pain on attempted upward or downward gaze. Pain was the most common sign of disease activity. Conjunctival hyperemia, which was found in 20 patients $(66.6 \%)$, was the second most common sign of disease activity. Two patients (6.6\%) with exophthalmos and diplopia underwent corticosteroid treatment.

The mean serum CysC concentrations in the GO and

Table 1. Demographic characteristics of the patient groups

\begin{tabular}{lcc} 
& GO group & Control group \\
\hline Age $(\mathrm{yr})$ & $36.1 \pm 18.4$ & $34 \pm 10.1$ \\
Sex & & \\
$\quad$ Male & $17(56.6)$ & $16(53.3)$ \\
Female & $13(43.3)$ & $14(46.6)$ \\
\hline
\end{tabular}

Values are presented as mean \pm standard deviation or number (\%). $\mathrm{GO}=$ Graves' ophthalmopathy.

Table 2. Serum CysC, TSH, FT4, and FT3 concentrations of the GO patients and control groups

\begin{tabular}{lccc}
\hline & $\begin{array}{c}\text { GO group } \\
(\mathrm{n}=30)\end{array}$ & $\begin{array}{c}\text { Control group } \\
(\mathrm{n}=30)\end{array}$ & $p_{\text {-value }}{ }^{*}$ \\
\hline CysC $(\mathrm{mg} / \mathrm{L})$ & $1.04 \pm 0.36$ & $0.74 \pm 0.09$ & $<0.001$ \\
TSH $(\mathrm{IU} / \mathrm{mL})$ & $1.76 \pm 0.74$ & $2.73 \pm 0.78$ & 0.954 \\
FT4 $(\mathrm{ng} / \mathrm{dL})$ & $1.60 \pm 0.45$ & $1.40 \pm 0.22$ & $<0.001$ \\
FT3 $(\mathrm{pg} / \mathrm{mL})$ & $4.06 \pm 1.10$ & $3.73 \pm 0.76$ & 0.008 \\
\hline
\end{tabular}

Values are presented as mean \pm standard deviation.

CysC $=$ cystatin $\mathrm{C}$; TSH $=$ thyroid-stimulating hormone; FT4 = free thyroxine; FT3 = free triiodothyronine; $\mathrm{GO}=$ Graves' ophthalmopathy.

"Student's $t$-test. 
Table 3. Serum CysC, TSH, FT4, and FT3 concentrations of hyperthyroid and euthyroid GO patients

\begin{tabular}{lccr}
\hline & $\begin{array}{c}\text { Hyperthyroid } \\
\text { GO patients } \\
(\mathrm{n}=15)\end{array}$ & $\begin{array}{c}\text { Euthyroid GO } \\
\text { patients } \\
(\mathrm{n}=15)\end{array}$ & $p$-value \\
\hline CysC $(\mathrm{mg} / \mathrm{L})$ & $1.35 \pm 0.22$ & $0.72 \pm 0.13$ & 0.001 \\
TSH $(\mathrm{IU} / \mathrm{mL})$ & $1.25 \pm 0.13$ & $2.27 \pm 0.75$ & $<0.001$ \\
FT4 $(\mathrm{ng} / \mathrm{dL})$ & $1.99 \pm 0.21$ & $1.21 \pm 0.24$ & 0.161 \\
FT3 $(\mathrm{pg} / \mathrm{mL})$ & $5.03 \pm 0.14$ & $3.09 \pm 0.69$ & $<0.001$ \\
\hline
\end{tabular}

Values are presented as mean \pm standard deviation.

CysC $=$ cystatin $\mathrm{C}$; TSH $=$ thyroid-stimulating hormone; FT4 = free thyroxine; FT3 = free triiodothyronine; $\mathrm{GO}=$ Graves' ophthalmopathy.

"Student's $t$-test.

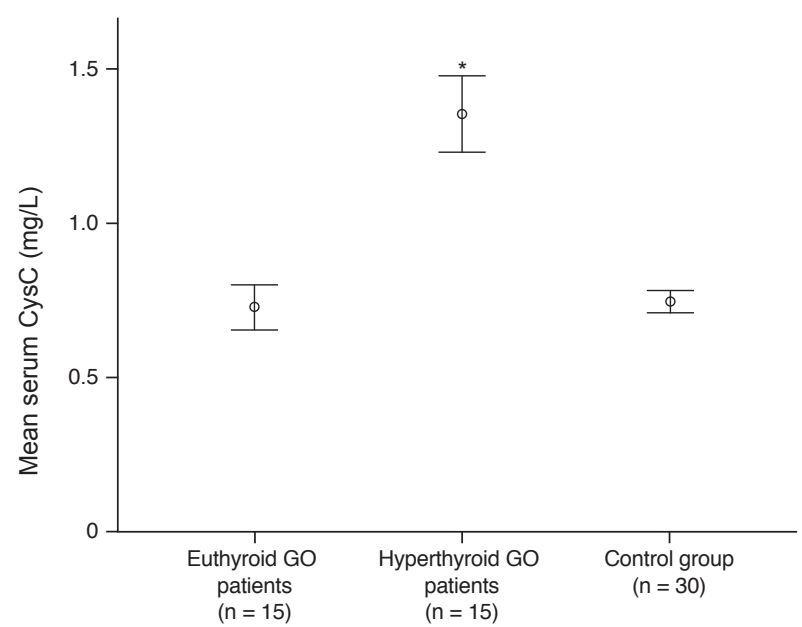

Fig. 1. The mean serum cystain $\mathrm{C}(\mathrm{CysC})$ concentrations of euthyroid and hyperthyroid Graves' ophthalmopathy (GO) patients and controls. "Kruskal-Wallis test.

control groups were $1.04 \pm 0.36$ and $0.74 \pm 0.09 \mathrm{mg} / \mathrm{L}$, respectively. The serum CysC concentrations were significantly higher in patients with GO than in the control subjects $(p<0.001)$. Moreover, there were statistically significant differences in the FT4 and FT3 concentrations between the GO and control groups (Table 2). Furthermore, there were statistically significant differences in the serum CysC, TSH, and FT3 concentrations between the hyperthyroid and euthyroid subgroups (Table 3). The mean serum CysC concentrations in the hyperthyroid and euthyroid subgroups were $1.35 \pm 0.22$ and $0.72 \pm 0.13 \mathrm{mg} / \mathrm{L}$, respectively, showing a significant difference $(p=0.001)$. Serum CysC concentrations in healthy subjects were significantly lower than those of hyperthyroid patients $(p<$
$0.001)$ but similar to those of euthyroid patients $(p=1.00)$ (Fig. 1).

\section{Discussion}

Thyroid dysfunction affects serum CysC concentration $[15,16]$, but the underlying mechanisms are unclear. Kotajima et al. [13] reported a positive correlation between serum $\mathrm{Cys} \mathrm{C}$ concentration and thyroid hormones in patients with thyroid disorders and suggested that the underlying mechanisms may be elevation of the serum transforming growth factor $\beta 1$ concentrations and direct stimulatory effects of 3,3',5-triiodo-L-thyronine (T3) and transforming growth factor $\beta 1$ on CysC production. Similarly, Schmid et al. [17] suggested that elevated T3 concentrations may directly increase $\mathrm{CysC}$ production.

Fricker et al. [11] studied serum CysC concentrations in nine patients with hypothyroidism and in 13 patients with hyperthyroidism, and they reported that $\mathrm{Cys} C$ concentrations are significantly lower in hypothyroidism and significantly higher in hyperthyroidism compared to euthyroid status. They explained these changes through a direct effect of thyroid hormones on CysC production.

Wiesli et al. [18] studied serum CysC concentration in 26 patients with subclinical hypothyroidism and in 14 patients with subclinical hyperthyroidism and, when CysC concentration was compared within the same individual at different time points, hypothyroid status was associated with lower $\mathrm{Cys} C$ and hyperthyroid status with higher $\mathrm{CysC}$ compared to euthyroid status in the same individual. They concluded that mild thyroid dysfunction significantly changes CysC concentration.

In contrast, Ye et al. [19] reported that both subclinical hypothyroidism and hyperthyroidism are associated with increased serum CysC concentrations in men. They explained this discrepancy with differences in experimental design.

In the present study, serum CysC concentrations in patients with GO were significantly higher than those of control subjects. Our study was different from previous studies in that it was performed in patients who had GD and ophthalmopathy. Moreover, in previous studies, the control groups consisted of hypothyroid patients or the same individuals in euthyroid status, but our control group consisted of healthy subjects. In our study, CysC concentrations were significantly higher in hyperthyroid patients com- 
pared to euthyroid patients. Compared to healthy subjects, serum CysC concentrations were significantly higher in hyperthyroid patients and not significantly different in euthyroid patients. Our results suggest that high serum CysC concentrations in patients with GO are mainly caused by the thyroid hormones. Our explanation for the cause of high serum $\mathrm{Cys} C$ concentration is consistent with previous studies, which reported that serum CysC concentration increases in hyperthyroidism $[11,13,18]$.

Our study had some limitations. First, the number of cases included in the study was low. Second, our study group consisted of patients with GO having a hyperthyroid or euthyroid status but none having a hypothyroid status. Therefore, hypothyroid and hyperthyroid patients with GO could not be compared. Furthermore, glucocorticoid therapy was not considered in the interpretation of our results. In some previous studies, serum CysC concentrations were affected by methylprednisolone or prednisone [20,21]. Therefore, both acute and long-term glucocorticoid administration should be considered in the interpretation of serum CysC concentration.

In conclusion, this is the first study to evaluate serum CysC levels in patients with GO. The results of the current study showed increased serum CysC concentration in hyperthyroid patients with GO. Since hyperthyroid patients with GO had higher CysC concentrations than euthyroid patients with GO, we believe that increased serum CysC concentrations are mainly caused by thyroid hormones. However, the mechanism remains to be elucidated. Future studies are needed that will include hyperthyroid patients with GO to investigate the possible use of serum CysC concentration as a marker in follow-up. Further evaluation of serum CysC concentration in a larger cohort of patients with GO is necessary to confirm our results.

\section{Conflict of Interest}

No potential conflict of interest relevant to this article was reported.

\section{References}

1. Jinguji M, Nakajo M, Nakajo M, et al. Thymic involution after radioiodine therapy for Graves disease: relationships with serum thyroid hormones and TRAb. J Endocr Soc 2017;1:852-60.

2. Kamboj A, Lause M, Kumar P. Ophthalmic manifestations of endocrine disorders-endocrinology and the eye. Transl Pediatr 2017;6:286-99.

3. Xiong $\mathrm{H}, \mathrm{Wu} \mathrm{M}, \mathrm{Yi} \mathrm{H}$, et al. Genetic associations of the thyroid stimulating hormone receptor gene with Graves diseases and Graves ophthalmopathy: a meta-analysis. Sci Rep 2016;6:30356.

4. Xia N, Ye X, Hu X, et al. Simultaneous induction of Graves' hyperthyroidism and Graves' ophthalmopathy by TSHR genetic immunization in BALB/c mice. PLoS One 2017;12:e174260.

5. Xu L, Li L, Xie C, et al. Thickness of extraocular muscle and orbital fat in MRI predicts response to glucocorticoid therapy in Graves' ophthalmopathy. Int J Endocrinol 2017;2017:3196059.

6. Li YJ, Luo Y, He WM, et al. Clinical outcomes of graves' ophthalmopathy treated with intensity modulated radiation therapy. Radiat Oncol 2017;12:171.

7. Khatavi F, Nasrollahi K, Zandi A, et al. A promising modified procedure for upper eyelid retraction-associated Graves' ophthalmopathy: transconjunctival lateral levator aponeurectomy. Med Hypothesis Discov Innov Ophthalmol 2017;6:44-8.

8. Hao HT, Wang Y, Wang X, et al. Treatment of Graves' ophthalmopathy with an in-house Phosphorus-32 source: initial clinical observations. Exp Ther Med 2017;14:2795800.

9. Sabini E, Leo M, Mazzi B, et al. Does Graves' orbitopathy ever disappear?: answers to an old question. Eur Thyroid $J$ 2017;6:263-70.

10. De Souza V, Hadj-Aissa A, Dolomanova O, et al. Creatinine- versus cystatine $\mathrm{C}$-based equations in assessing the renal function of candidates for liver transplantation with cirrhosis. Hepatology 2014;59:1522-31.

11. Fricker M, Wiesli P, Brandle M, et al. Impact of thyroid dysfunction on serum cystatin C. Kidney Int 2003;63:19447.

12. Ozden TA, Tekerek H, Bas F, Darendeliler F. Effect of hypo-and euthyroid status on serum cystatin C levels. J Clin Res Pediatr Endocrinol 2010;2:155-8.

13. Kotajima N, Yanagawa Y, Aoki T, et al. Influence of thyroid hormones and transforming growth factor- $\beta 1$ on cystatin C concentrations. J Int Med Res 2010;38:1365-73.

14. Bartalena L, Baldeschi L, Boboridis K, et al. The 2016 European Thyroid Association/European Group on Graves' 
Orbitopathy Guidelines for the Management of Graves' Orbitopathy. Eur Thyroid J 2016;5:9-26.

15. Jayagopal V, Keevil BG, Atkin SL, et al. Paradoxical changes in cystatin $\mathrm{C}$ and serum creatinine in patients with hypo- and hyperthyroidism. Clin Chem 2003;49:680-1.

16. Den Hollander JG, Wulkan RW, Mantel MJ, Berghout A. Is cystatin $\mathrm{C}$ a marker of glomerular filtration rate in thyroid dysfunction? Clin Chem 2003;49:1558-9.

17. Schmid C, Ghirlanda-Keller C, Zwimpfer C, Zoidis E. Triiodothyronine stimulates cystatin $\mathrm{C}$ production in bone cells. Biochem Biophys Res Commun 2012;419:425-30.

18. Wiesli P, Schwegler B, Spinas GA, Schmid C. Serum cys- tatin $\mathrm{C}$ is sensitive to small changes in thyroid function. Clin Chim Acta 2003;338:87-90.

19. Ye Y, Gai X, Xie H, et al. Impact of thyroid function on serum cystatin $\mathrm{C}$ and estimated glomerular filtration rate: a cross-sectional study. Endocr Pract 2013;19:397-403.

20. Cimerman N, Brguljan PM, Krasovec M, et al. Serum cystatin $\mathrm{C}$, a potent inhibitor of cysteine proteinases, is elevated in asthmatic patients. Clin Chim Acta 2000;300:83-95.

21. Risch L, Herklotz R, Blumberg A, Huber AR. Effects of glucocorticoid immunosuppression on serum cystatin $\mathrm{C}$ concentrations in renal transplant patients. Clin Chem 2001;47:2055-9. 